\title{
Kaiso represses the expression of glucocorticoid receptor via a methylation-dependent mechanism and attenuates the anti-apoptotic activity of glucocorticoids in breast cancer cells
}

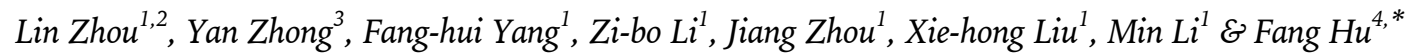 \\ ${ }^{1}$ Department of Clinical Biochemistry, Clinical Medicine Laboratory, ${ }^{2}$ Department of Anatomy Histology and Embryology, Institute of \\ Neuroscience, Changsha Medical University, Changsha, Hunan 410219, ${ }^{3}$ Department of Gynaecology and Obstetrics, ${ }^{4}$ Metabolic Syndrome \\ Research Center, The Second Xiangya Hospital of Central South University, Changsha, Hunan 410011, China
}

\begin{abstract}
Kaiso is a Pox Virus and Zinc Finger (POZ-ZF) transcription factor with bi-modal DNA-binding specificity. Here, we demonstrated that Kaiso expression is inversely correlated with glucocorticoid receptor (GR) expression in breast carcinomas. Knockdown of Kaiso increased GR expression, while overexpression of Kaiso inhibited GR expression in breast cancer cells. Furthermore, Kaiso repressed GR proximal promoter-reporter activity in a dose-dependent manner. Remarkably, ChIP experiments demonstrated that endogenous Kaiso was associated with the GR promoter sequence in a methylation-dependent manner. Since glucocorticoids inhibit chemotherapyinduced apoptosis and have been widely used as a co-treatment of patients with breast cancer, we assessed the role of Kasio in GR-mediated anti-apoptotic effects. We found that overexpression of Kaiso attenuated the anti-apoptotic effects of glucocorticoids in breast cancer cells. Our findings suggest that GR is a putative target gene of Kaiso and suggest Kaiso to be a potential therapeutic target in GC-combination chemotherapy in breast cancer. [BMB Reports 2016; 49(3): 167-172]
\end{abstract}

\section{INTRODUCTION}

Kaiso is a POZ-ZF transcription factor originally identified as a binding partner of the cell adhesion molecule p120-catenin (1). It contains a POZ domain at its $\mathrm{N}$-terminal to mediate protein-protein interactions and zinc finger motifs at its C-terminal to mediate DNA binding. Kaiso can either bind DNA in a sequence-specific manner via the consensus Kaiso binding site (KBD) CTGCNA or directly interact with methylated CpG-di-

*Corresponding author. Tel: +(011)86 731 88602865; Fax: +(011) 86731 84591661; E-mail: hufanghf@yeah.net

http://dx.doi.org/10.5483/BMBRep.2016.49.3.151

Received 16 July 2015, Revised 13 August 2015, Accepted 1 October 2015

Keywords: Breast cancer, Epigenetics, Glucocorticoid receptor, Kaiso nucleotides to regulate its target gene expression (2). Kaiso functions primarily as a transcriptional repressor (3-5). In breast cancer, nuclear Kaiso expression was associated with poor prognostic factors and invasive breast carcinoma $(6,7)$. It has been reported that Kaiso binds directly to methylated sequences in the E-cadherin promoter to promote the epithelialmesenchymal transition in breast cancer (7). However, the downstream target genes of Kaiso in breast cancer remain to be further characterized.

The glucocorticoid receptor (GR) belongs to the nuclear receptor superfamily of transcription factors (8). Glucocorticoids are the main ligand for GR, in addition to other ligands including progesterone and dehydroepiandrosterone. Activation of GR by its ligands induces the expression of many genes involved in anti-inflammatory signaling pathways(8). GR also exerts an anti-apoptotic effect in breast cancer through activation of NF-kB or AP-1 $(9,10)$. The expression of GR is observed at all stages from normal to cancerous breast tissue and its level generally decreases during cancer progression (11). A study of breast carcinomas found that the GR proximal promoter was methylated in $15 \%$ of tumor samples, while no GR promoter methylation was observed in normal breast tissues (12). Activation of GR in preclinical human breast cancer models exerted an anti-proliferative effect, while deregulation of the GR signaling pathway promoted tumorigenesis (11). Thus, GR may function as a tumor suppressor in tumor development $(13,14)$.

Although the GR promoter has been reported to be methylated in multiple human cancers, including breast cancer (12, $15,16)$, little is known about its transcriptional regulation by other DNA-binding factors. In this study, we demonstrate that Kaiso represses GR expression by binding to the GR proximal promoter in a DNA methylation-dependent manner. Our study further shows that overexpression of Kaiso attenuates the anti-apoptotic activity of glucocorticoids in breast cancer cells.

ISSN: 1976-670X (electronic edition)

Copyright (C) 2016 by the The Korean Society for Biochemistry and Molecular Biology

(c) This is an open-access article distributed under the terms of the Creative Commons Attribution Non-Commercial License (http://creativecommons.org/licenses/by-nc/4.0) which permits unrestricted non-commercial use, distribution, and reproduction in any medium, provided the original work is properly cited. 


\section{MATERIAL AND METHODS}

\section{Reagents}

Antibodies to Kaiso (6F/6F8), Glucocorticoid Receptor (BuGR2), phosphor-p65 (ab86299), p65 (ab16502), and Sgk-1 (ab59337) were purchased from Abcam (Cambridge, MA, USA). Antibody to $\beta$-actin was purchased from Santa Cruz Biotechnology (Santa Cruz, CA, USA). Human TNF- $\alpha$ was purchased from R\&D Systems (MN, USA). Other reagents were purchased from Sigma-Aldrich (St. Louis, MO, USA).

\section{Constructs}

Human Kaiso and GR full-length sequences were amplified from reverse transcribed cDNA of MCF-7 cells. Then, the Kaiso and GR sequences were cloned into the pCMV-Tag 2A (Agilent Technology, USA) and pCMV-Myc (Clonetech, USA) mammalian expression vectors, respectively. DNA sequencing confirmed the sequence fidelity. A pair of PCR primers was used to amplify the region of the $3 \mathrm{~kb}$ GR proximal promoter between -1668 and -4667 . PCR products were ligated into a T/A cloning vector and sequenced to confirm sequence fidelity. Then, the $3 \mathrm{~kb} G R$ proximal promoter fragment was subcloned into the pGL3-basic plasmid (Promega, USA).

\section{Cell lines}

The human breast cancer cell lines BT-20, MDA-MB-231, MCF-7 and T47D were purchased from ATCC and cultured at $37^{\circ} \mathrm{C}$ with $5 \% \mathrm{CO}_{2}$ in DMEM Medium (Invitrogen, USA) supplemented with $10 \%$ fetal bovine serum, 100 units $/ \mathrm{ml}$ penicillin G, $100 \mu \mathrm{g} / \mathrm{ml}$ streptomycin, and $2 \mathrm{mM}$ L-glutamine.

\section{Western blotting}

Cells were lysed in $\mathrm{M} 2$ lysis buffer $(150 \mathrm{mM} \mathrm{NaCl}, 50 \mathrm{mM}$ Tris-Cl (pH 8.0), 5 mM EDTA, $1 \%$ Nonidet P-40) containing a protease inhibitor mixture (Roche Applied Science) and a phosphatase inhibitor mixture (Sigma, MO, USA). An equal amount of total protein was subjected to SDS-PAGE analysis and immunoblotting using the appropriate antibodies.

\section{Transient transfection, luciferase assay and siRNA knockdown}

Following the manufacturer's instructions, plasmids were transfected into cells using Lipofectamine 2000 (Invitrogen, USA). For luciferase assay, $0.8 \mu \mathrm{g}$ of reporter plasmids plus $0.05 \mu \mathrm{g}$ of pCMV-LacZ vector and FLAG-Kaiso or empty vector were transfected into cells using Lipofectamine 2000. At $24 \mathrm{~h}$ post-transfection, cells were analyzed for luciferase activity. Luciferase assays were performed using the luciferase assay system (Promega); $\beta$-galactosidase activity was used as an internal control. Each experiment was conducted in at least triplicate.

For siRNA treatments, T47D cells were plated in a $3.5 \mathrm{~cm}$ dish and transfected with 50 pm Kaiso or non-targeting siRNA (Human ONTARGETplus siRNA pools of four oligos, Dharmacon,
USA) using Lipofectamine RNAi Max (Invitrogen, USA). After transfection for $48 \mathrm{~h}$, cells were subjected to western blotting.

\section{ChIP Assay}

The ChIP assay was performed using the ChIP assay kit from Upstate Biotechnologies, Inc. (Lake Placid, NY). Cells $(1 \times$ $10^{6}$ ) were fixed with $1 \%$ formaldehyde for 10 min, chromatin was fragmented by sonication to yield fragments between 200 and $1,000 \mathrm{bp}$, and $10 \mu \mathrm{l}$ of the chromatin solution were saved as input. One microgram of antibody or control immunoglobulin $\mathrm{G}$ was added to tubes containing $900 \mu \mathrm{l}$ of chromatin solution. After incubation, the antibody complexes were captured with protein A-agarose beads and subjected to serial washes. The chromatin fraction was further extracted, and the cross-linked chromatin was reversed at $65^{\circ} \mathrm{C}$ in the presence of $200 \mathrm{mM} \mathrm{NaCl}$. The DNA was then purified using chloroform/isoamyl alcohol. The PCR primers used to amplify the GR proximal promoter regions were as follows: P1 set, fwd: 5'-GCAACGCACA GAGTCGAGGG C-3', rev: 5'-CGCCCAATGT GCTCACACTCG-3', P2 set, fwd: 5'-CCCCGGGCCCAAAGTACGTATGCG-3', rev: 5'-GCGGCTGAGCTGCGTGAGTGG-3', P3 set, fwd: 5'-CGAGTGTGTG CGCGCCGT-3', rev: 5'-CGGCGTCTCC TTCCACCCAC-3', P4 set, fwd: 5'-CCGCCGCGGG AGCCTACAAA-3', rev: 5'-ACGAAAACGG GTGTCGGGCG-3. PCR products were then run on an agarose gel, stained with ethidium bromide, and photographed.

\section{Real-time PCR}

Tumor and normal breast clinical tissue samples were obtained from the second hospital affiliated to XiangYa, Changsha, Hunan, China. This study was approved by the Research Ethics Committee of Second Xiangya Hospital, Hunan, China. Informed consent was obtained from all of the patients. TRIzol reagent (Invitrogen, USA) was used to isolate total RNA. cDNA was prepared using reverse transcriptase SuperScript III (Invitrogen, USA) and $2 \mu \mathrm{g}$ of total RNA. Then, $2 \mu \mathrm{l}$ of cDNA were added to an $18 \mu \mathrm{l}$ PCR assay mixture containing $0.5 \mathrm{M}$ each probe and $10 \mu$ l Taqman Master Mix (Life Technologies, USA). PCR was conducted with the MyiQ Real-Time PCR Detection System (Bio-Rad, USA). Taqman probes for Kaiso (Assay ID: Hs.PT.58.3381372), GR (Assay ID: Hs.PT.58.27480377), ICAM-1 (Assay ID: Hs. 00164932), IAP-2 (Assay ID: Hs. 00985029) and Actin (Assay ID: Hs.PT.56a.21538384) were purchased from Life Technologies. The threshold cycle number for each gene was normalized to that of $\beta$-actin, and the resulting value was converted to a linear scale. All assays were performed in at least triplicate from independent RNA preparations.

\section{Statistical analysis}

Data were analyzed using the SPSS (version 20.0; IBM Corporation, Armonk, NY, USA) software. Results are expressed as means \pm S.D. and are representative of at least three independent experiments. The two-sample t-test was used to de- 
termine the significance of differences in the means of two columns. A P value less than 0.05 was regarded as indicative of statistical significance.

\section{RESULTS}

\section{Kaiso expression inversely correlates with GR expression in breast carcinomas}

To study the functional relationship between the transcription factor Kaiso and GR in breast cancer, we first examined the mRNA levels of Kaiso and GR in human breast carcinomas. A total of 32 breast carcinoma samples were collected, which comprised 26 infiltrating ductal carcinomas (IDC), 4 ductal carcinomas in situ (DCIS) and 2 invasive lobular carcinomas (ILC). Interestingly, we found that Kaiso expression was inversely correlated with GR expression in breast cancer samples (Fig. 1A). To confirm this observation, immunoblotting analysis was performed using a further seven IDCs and one normal breast tissue. As shown in Fig. 1B, tumor samples with

\section{A}

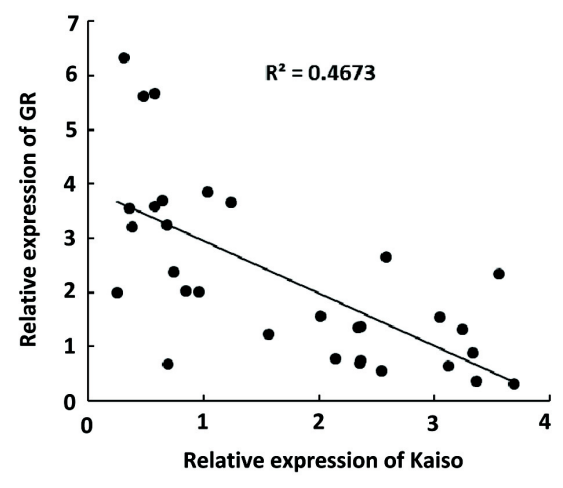

B

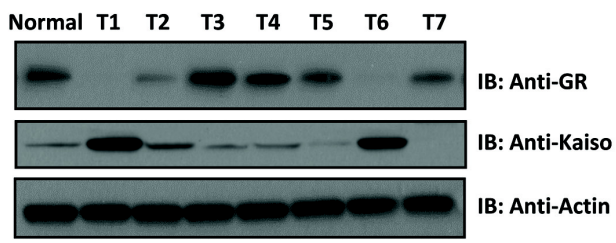

Fig. 1. Kaiso expression is inversely correlated with GR expression in breast carcinomas. (A) GR and Kaiso mRNA levels in 32 breast cancer samples were determined by Taqman assays and the correlation between GR expression and Kaiso was determined by Pearson's correlation analysis. (B) Western blotting analysis of GR and Kaiso expression in one human normal breast tissue and seven human breast carcinomas. T: Tumor. lower expression of Kaiso showed elevated expression of GR (Fig. 1B).

\section{Kaiso represses GR expression in breast cancer cells}

As Kaiso expression was inversely correlated with GR expression in breast tumor samples, we then determined whether Kaiso negatively regulates the expression of GR in breast cancer. We found that Kaiso expression was also inversely associated with GR expression in various breast cancer cell lines (Fig. 2A). Since T47D cells showed the highest Kaiso expression among all breast cancer cell lines, we then used siRNA to knockdown Kaiso expression in the cells to investigate the regulatory effects of Kaiso on GR expression. As shown in Fig. 2B, knockdown of Kaiso increased GR expression in T47D cells. Furthermore, overexpression of Kaiso in breast cancer cells inhibited GR expression (Fig. 2C and D). Since Kaiso is a transcriptional repressor, we then employed a
A

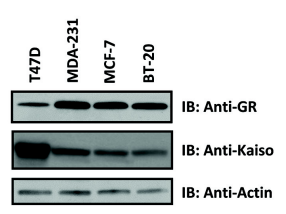

C
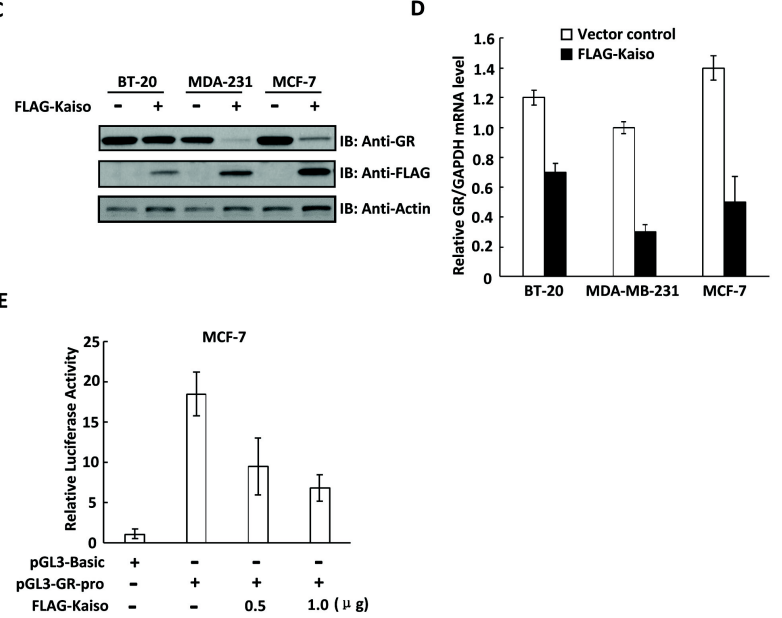

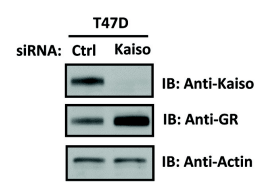

Fig. 2. Kaiso represses GR expression in breast cancer cell lines. (A) Kaiso and GR expression levels in breast cancer cell lines were determined by western blotting. (B) Kaiso and GR expression levels were determined by western blotting in siRNA control or Kaiso-knockdown T47D cells. (C) Overexpression of Kaiso in breast cancer cell lines and GR and Kaiso expression levels were determined by western blotting. (D) Kaiso mRNA levels in vector control or Kaiso-overexpressing breast cancer cell lines were determined by real-time PCR. (E) Overexpression of Kaiso in MCF-7 cells decreased the luciferase activity of the GR promoter-reporter in a dose-dependent manner. 
GR promoter luciferase assay to test whether Kaiso influenced the transcriptional activity of the GR promoter. We found that overexpression of Kaiso decreased the promoter activity of GR
A

C

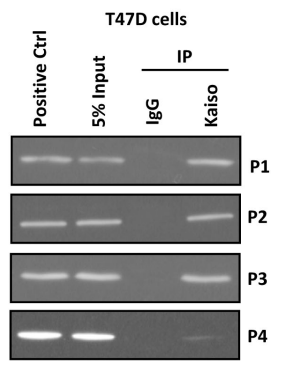

E

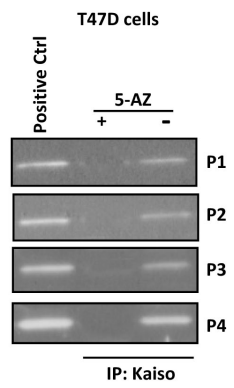

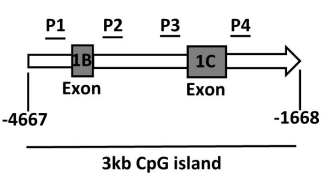

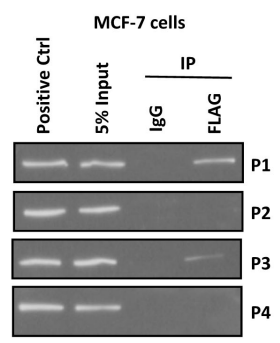

D

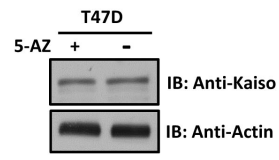

Fig. 3. Kaiso binds to the GR promoter sequence via a methylCpG-dependent mechanism. (A) Schematic diagram of the GR proximal promoter. The two main alternative exons are labeled $1 \mathrm{~B}$ and $1 \mathrm{C}$. Relative to the translation start site, the P1 primer set amplifies the 283 bp region from -4046 to 3764 , the P2 primer set amplifies the 209 bp region from -3549 to -3339 , the P3 primer set amplifies the 204 bp region from -2868 to -2665 , and the P4 primer set amplifies the 298 bp region from -2294 to -1997. (B) MCF-7 cells were induced to overexpress FLAG-fused Kaiso. Chromatin was immunoprecipitated using an antibody against FLAG or (C) Kaiso in T47D cells. Anti-IgG antibody was used as a negative control. Sets of primers were used to amplify the various GR promoter regions from immunoprecipitated chromatin. The GR promoter plasmid was used as a positive control for PCR. (D) T47D cells were treated with 5-AZ, and Kaiso expression was determined by western blotting. (E) ChIP analysis of the various GR promoter regions in T47D cells. Data shown represent results of two independent experiments. in a dose-dependent manner (Fig. 2E). Collectively, our data suggested that Kaiso inhibited GR expression and its promoter activity in breast cancer cells.

\section{Kaiso binds to the GR promoter sequence via a methyl-CpG-dependent mechanism}

Since Kaiso can bind DNA in both a sequence- and methyl-CpG-specific manner, we then determined whether Kaiso repressed GR promoter activity by directly interacting with its promoter sequence. The GR proximal promoter is located in a $3 \mathrm{~kb} \mathrm{CpG}$ island upstream of the translation start site and contains seven alternative first exon sequences, including exon $1 \mathrm{~B}$ and $1 \mathrm{C}$ (gray boxes) (Fig. 3A). In breast cancer cells, expression of GR transcripts originated from either promoter 1B or promoter $1 \mathrm{C}(12)$. Since there is no Kaiso-binding site in the GR proximal promoter region and the GR promoter is methylated in breast cancer cells (12), we then determined whether Kaiso could bind DNA in a methyl-CpG-dependent manner. Four sets of primers specific for CpG islands in the GR promoter were designed and named P1 to P4 (Fig. 3A). We first overexpressed FLAG-Kaiso in MCF-7 cells and found that overexpressed Kaiso associated with the P1 and P3 promoter regions (Fig. 3B). To confirm this result, we used a Kaiso-specific monoclonal antibody $6 \mathrm{~F}$ to perform a ChIP analysis in T47D cells, which express a high level of Kaiso. We found that Kaiso bound directly to all four promoter sequences in different regions (Fig 3C). To test whether Kaiso bound to the GR promoter in a methylation-dependent manner, we treated T47D cells with the DNA demethylation reagent 5-AZ for $48 \mathrm{~h}$. Treatment with 5-AZ did not change Kaiso expression (Fig. 3D); however, 5-AZ abolished binding of Kaiso to the GR promoter (Fig. 3E), suggesting that Kaiso binds to the GR promoter via a methyl-CpG-dependent mechanism.

\section{Kaiso represses the anti-apoptotic effect of GR signaling in breast cancer cells}

It has been reported that glucocorticoids (GCs) initiate an anti-apoptosis signaling pathway and protect breast cancer cells from apoptosis $(17,18)$. To study the role of Kaiso in the anti-apoptotic effects of the GR signaling pathway, we overexpressed Kaiso in MCF-7 cells and then induced apoptosis by growth factor deprivation (Fig. 4A) or TNF- $\alpha$ treatment (Fig. $4 B$ ). We found that overexpression of Kaiso significantly attenuated the anti-apoptotic effects of DEX (Fig. 4A and B). To determine whether Kaiso antagonized cell survival specifically through GR, we co-expressed GR in Kaiso-overexpressing MCF-7 cells (Fig. 4C), and found GR overexpression restored the anti-apoptotic effect of DEX (Fig 4D). Previous studies demonstrated that GC activated the NF- $\mathrm{kB}$ signaling pathway in breast cancer cells and induced expression of several anti-apoptosis genes, including sgk-1, bcl-xL and bak (17-20). To further investigate the role of Kaiso in regulation of the GR signaling pathway, we then analyzed p65 phosphorylation and sgk-1 expression in Kaiso-overexpressing cells. Consistent with 
A

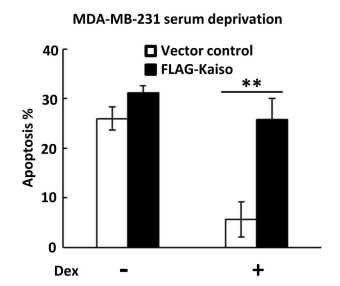

C

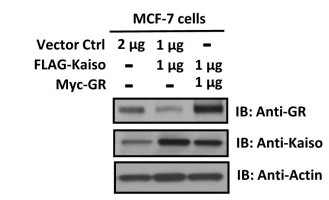

E

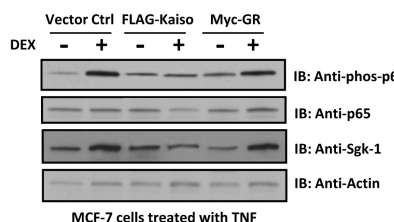

MCF-7 cells treated with TNF

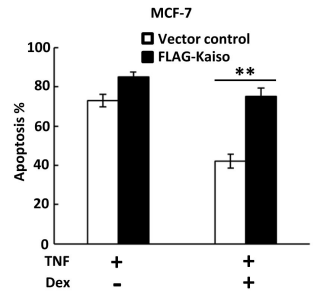

D

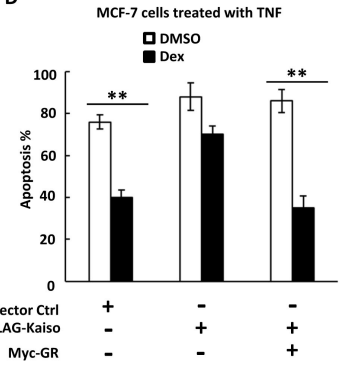

$\mathbf{F}$

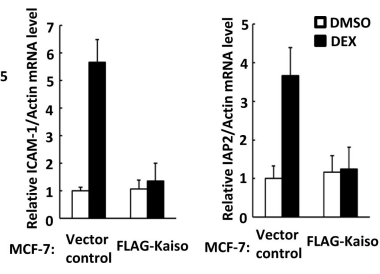

Fig. 4. Kaiso attenuates the anti-apoptotic effect of GR signaling in breast cancer cells. (A) MDA-MB-231 cells were transfected with vector control or FLAG-Kaiso and then serum starved for $72 \mathrm{~h}$ in the presence or absence of dexamethasone $\left(10^{6} \mathrm{M}\right)$. The percentage of apoptotic cells was determined by Annexin $\mathrm{V}$ staining. (B) MCF-7 cells were transfected with vector control or FLAG-Kaiso and then treated with TNF- $\alpha(10 \mathrm{ng} / \mathrm{ml})$ for $48 \mathrm{~h}$ in the presence or absence of dexamethasone. The percentage of apoptotic cells was determined by Annexin $\mathrm{V}$ staining. (C) MCF-7 cells were transfected with vector control, FLAG-Kaiso or Myc-GR as indicated, and the expression levels of Kaiso and GR were determined by western blotting. (D) The cells used in (C) were treated with TNF- $\alpha(10 \mathrm{ng} / \mathrm{ml})$ for $48 \mathrm{~h}$ in the presence or absence of dexamethasone. The percentage of apoptotic cells was determined by Annexin $\mathrm{V}$ staining. (E) p65 phosphorylation and Sgk-1 expression in the MCF-7 cells used in (C) were determined by western blotting. (F) ICAM-1 and CIAP2 expression levels in MCF-7 cells were determined by real-time PCR. Data shown represent the results of three independent experiments. ${ }^{* *} \mathrm{P}<0.01$.

previous studies, DEX induced phosphorylation of p65 and sgk-1 expression in control cells, while overexpression of Kaiso inhibited phosphorylation of p65 and sgk-1 expression (Fig. 4E). Importantly, co-expression of GR with Kaiso restored the DEX-induced phosphorylation of p65 and sgk-1 expression, suggesting a regulatory role for Kaiso in the GR signaling pathway by targeting GR expression (Fig. 4E). Furthermore, overexpression of Kaiso also inhibited expression of $\mathrm{NF}-\kappa \mathrm{B}$-induced

anti-apoptotic target genes, including ICAM-1 and ClAP2 (Fig. 4F).

\section{DISCUSSION}

As a stress hormone (glucocorticoid) receptor, GR plays an important role in breast cancer cell biology (11). Since GR is the key mediator of cellular responses to GCs, its expression must be strictly regulated. In this study, we found that expression of the POZ-ZF transcription factor Kaiso was inversely correlated with GR expression in most invasive ductal carcinomas (IDC). The nuclear expression of Kaiso in IDC has been shown to be related to poor prognostic factors, including high grade, poor differentiation and metastasis $(6,7)$, while in ILC, most Kaiso proteins are localized in the cytoplasm and may function as a tumor suppressor (6). Since most tumor samples in this study were from IDC (80.1\% IDC samples, 26/32), our results most likely represented the nuclear function of Kaiso in breast cancer. As a transcriptional repressor, Kaiso could function as an oncogene to inhibit expression of tumor suppressor genes in multiple types of cancer. In colon cancer, Kaiso inhibited expression of the tumor suppressor gene CDKN2A by binding to its methylated promoter (21). It has been reported that Kaiso promotes breast cancer metastasis by binding to the methylated sequences in the E-cadherin promoter (7). However, Kaiso target genes in breast cancer still largely unknown. Our findings suggested that GR was a novel Kaiso target gene and supported an oncogenic role for Kaiso in breast cancer.

Kaiso is a dual-specificity transcription factor exhibiting DNA sequence- and methyl-CpG-specific transcriptional suppression activity. The human GR gene is regulated by two distinct promoter regions, the distal promoter and the proximal promoter (22). In breast cancer, the proximal promoter is predominantly responsible for GR expression (12). The proximal promoter has been shown to be methylated in gastric, lung, and breast cancers $(12,15)$. Loss of GR expression was associated with the methylation of its promoter and may contribute to tumorigenesis of breast cancer (12). We found that Kaiso was associated with the GR proximal promoter sequence in a methylation-dependent manner. Similarly, Kaiso regulates several tumor-associated genes, including $\mathrm{CDH} 1$ (E-cadherin) and CDKN2A, in methylation-dependent manner $(7,21,23)$. In addition, our results demonstrated that overexpression of Kaiso was associated with the P1 and P3 promoter regions in MCF-7 cells, while endogenous Kaiso was associated with all four promoter regions (P1 to P4) in T47D cells. The different affinity of Kaiso for GR promoter sequences may reveal the different levels of methylation of the GR promoter between MCF-7 and T47D cells. Thus, our data indicated GR to be a downstream target gene of Kaiso and may suggest a molecular mechanism for the transcriptional regulation of GR expression in breast cancer cells.

GCs have been used extensively as a co-medication with chemotherapy in breast cancer. These hormones have been shown to reduce inflammation and protect healthy tissues 
from cytotoxic side effects of the chemotherapy (11). Furthermore, the activation of GR by its ligands initiates an anti-apoptotic signal in several types of cancer, including breast cancer $(14,17,18)$. Our data demonstrated that Kaiso overexpression attenuated the anti-apoptotic effects of GR activation. The expression of Kaiso may have prognostic value in GC-combined chemotherapy in breast cancer. In future, it would be interesting to study the clinical role of Kaiso in GC-combined chemotherapy.

Our findings demonstrated that Kaiso inhibited GR expression by associating with the GR promoter sequence in a methylation-dependent manner in breast cancer. Importantly, a functional study suggested that Kaiso functioned as a suppressor in the GR signaling pathway. As GR plays important roles in breast cancer development and chemotherapy, our study suggests Kaiso to be a potential therapeutic target in breast cancer.

\section{ACKNOWLEDGEMENTS}

This study was supported by the Outstanding Youth Research Foundation of Education Bureau of Hunan Province, China (No. 2011B017) and the National Natural Science Foundation of China (Grant No. 81300503).

\section{REFERENCES}

1. Daniel JM and Reynolds AB (1999) The catenin p120(ctn) interacts with Kaiso, a novel BTB/POZ domain zinc finger transcription factor. Mol Cell Biol 19, 3614-3623

2. Daniel JM, Spring CM, Crawford HC, Reynolds $A B$ and Baig A (2002) The p120(ctn)-binding partner Kaiso is a bi-modal DNA-binding protein that recognizes both a sequence-specific consensus and methylated CpG dinucleotides. Nucleic Acids Res 30, 2911-2919

3. Ruzov A, Dunican DS, Prokhortchouk A et al. (2004) Kaiso is a genome-wide repressor of transcription that is essential for amphibian development. Development 131, 6185-6194

4. Donaldson NS, Nordgaard CL, Pierre CC et al (2010) Kaiso regulates Znf131-mediated transcriptional activation. Exp Cell Res 316, 1692-1705

5. Rodova M, Kelly KF, VanSaun M, Daniel JM and Werle MJ (2004) Regulation of the rapsyn promoter by kaiso and delta-catenin. Mol Cell Biol 24, 7188-7196

6. Vermeulen JF, van de Ven RA, Ercan C et al (2012) Nuclear Kaiso expression is associated with high grade and triple-negative invasive breast cancer. PLoS One 7, e37864

7. Jones J, Wang H, Karanam B et al (2014) Nuclear localization of Kaiso promotes the poorly differentiated phenotype and EMT in infiltrating ductal carcinomas. Clin Exp Metastasis 31, 497-510

8. Conzen SD (2008) Minireview: nuclear receptors and breast cancer. Mol Endocrinol 22, 2215-2228

9. Karmakar S, Jin Y and Nagaich AK (2013) Interaction of glucocorticoid receptor (GR) with estrogen receptor (ER) alpha and activator protein 1 (AP1) in dexamethasonemediated interference of ERalpha activity. J Biol Chem 288, 24020-24034

10. Ling J and Kumar R (2012) Crosstalk between NFkB and glucocorticoid signaling: a potential target of breast cancer therapy. Cancer Lett 322, 119-126

11. Vilasco M, Communal L, Mourra N, Courtin A, Forgez P and Gompel A (2011) Glucocorticoid receptor and breast cancer. Breast Cancer Res Treat 130, 1-10

12. Nesset KA, Perri AM and Mueller CR (2014) Frequent promoter hypermethylation and expression reduction of the glucocorticoid receptor gene in breast tumors. Epigenetics 9, 851-859

13. Yemelyanov A, Czwornog J, Chebotaev D et al (2007) Tumor suppressor activity of glucocorticoid receptor in the prostate. Oncogene 26, 1885-1896

14. Chebotaev D, Yemelyanov A, Zhu L, Lavker RM and Budunova I (2007) The tumor suppressor effect of the glucocorticoid receptor in skin is mediated via its effect on follicular epithelial stem cells. Oncogene 26, 3060-3068

15. Kay P, Schlossmacher G, Matthews L et al (2011) Loss of glucocorticoid receptor expression by DNA methylation prevents glucocorticoid induced apoptosis in human small cell lung cancer cells. PLoS One 6, e24839

16. Lind GE, Kleivi K, Meling Gl et al (2006) ADAMTS1, CRABP1, and NR3C1 identified as epigenetically deregulated genes in colorectal tumorigenesis. Cell Oncol 28, 259-272

17. Mikosz CA, Brickley DR, Sharkey MS, Moran TW and Conzen SD (2001) Glucocorticoid receptor-mediated protection from apoptosis is associated with induction of the serine/threonine survival kinase gene, sgk-1. J Biol Chem 276, 16649-16654

18. Machuca C, Mendoza-Milla C, Cordova E et al (2006) Dexamethasone protection from TNF-alpha-induced cell death in MCF-7 cells requires NF-kappaB and is independent from AKT. BMC Cell Biol 7, 9

19. Schorr K and Furth PA (2000) Induction of bcl-xL expression in mammary epithelial cells is glucocorticoid-dependent but not signal transducer and activator of transcription 5-dependent. Cancer Res 60, 5950-5953

20. Wu W, Chaudhuri S, Brickley DR, Pang D, Karrison T and Conzen SD (2004) Microarray analysis reveals glucocorticoid-regulated survival genes that are associated with inhibition of apoptosis in breast epithelial cells. Cancer Res 64, 1757-1764

21. Lopes EC, Valls E, Figueroa ME et al (2008) Kaiso contributes to DNA methylation-dependent silencing of tumor suppressor genes in colon cancer cell lines. Cancer Res 68, 7258-7263

22. Turner JD, Alt SR, Cao L et al (2010) Transcriptional control of the glucocorticoid receptor: CpG islands, epigenetics and more. Biochem Pharmacol 80, 1860-1868

23. Yoon HG, Chan DW, Reynolds AB, Qin J and Wong J (2003) N-CoR mediates DNA methylation-dependent repression through a methyl CpG binding protein Kaiso. Mol Cell 12, 723-734 